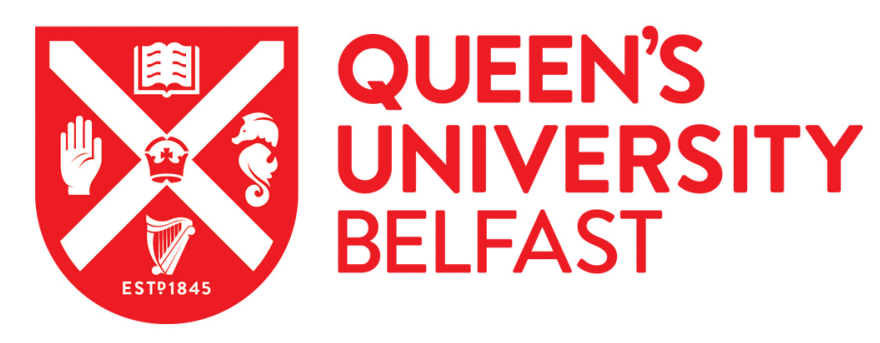

\title{
Lighting up the new order
}

Gregg, J. M. (2019). Lighting up the new order. Nature Materials, 18, 304-306. https://doi.org/10.1038/s41563019-0322-7

\section{Published in:}

Nature Materials

\section{Document Version:}

Peer reviewed version

\section{Queen's University Belfast - Research Portal:}

Link to publication record in Queen's University Belfast Research Portal

\section{Publisher rights}

Copyright 2019 Nature Research. This work is made available online in accordance with the publisher's policies. Please refer to any applicable terms of use of the publisher.

\section{General rights}

Copyright for the publications made accessible via the Queen's University Belfast Research Portal is retained by the author(s) and / or other copyright owners and it is a condition of accessing these publications that users recognise and abide by the legal requirements associated with these rights.

Take down policy

The Research Portal is Queen's institutional repository that provides access to Queen's research output. Every effort has been made to ensure that content in the Research Portal does not infringe any person's rights, or applicable UK laws. If you discover content in the Research Portal that you believe breaches copyright or violates any law, please contact openaccess@qub.ac.uk. 


\section{Lighting up the new order}

Irradiating a $\mathrm{PbTiO}_{3} / \mathrm{SrTiO}_{3}$ superlattice with ultrafast UV light pulses modifies elastic and electrostatic interactions, resulting in the formation of a stable complex 3D supercrystal.

\section{JM Gregg}

Finding good solutions, to satisfy multiple competing demands, is an artform that is hard to master. Yet nature does it all the time, often with startling and unexpected results. In the arid plains of Namibia and Australia, for example, the drive for plant-life to occupy all available space directly conflicts with the fact that only a limited biomass can be supported (due to low rainfall, as well as termite activity ${ }^{1,2}$ ). Instead of the optimum outcome being a uniform but sparse covering of vegetation, a grassland containing hexagonal arrays of circular discs of completely bare earth, termed "fairy rings", develops (figure 1a). These fairy rings are strikingly distributed, forming an ordered superstructure on scales vastly larger than the constituent components (grass and soil). Similarly, periodic superstructures are well known to naturally form in a variety of materials and are also the result of finding a balance between conflicting system needs (usually involving competing local and non-local energetics ${ }^{3,4}$ ). All such superstructures are interesting, but those seen by Stoica et al. ${ }^{5}$ in lead titanate / strontium titanate thin film multilayers, stand out as being particularly exotic. This is partly because the basic building blocks, from which the observed "supercrystals" form, are so new: the existence of ferroelectric dipolar vortices was first reported in $2016^{6}$. It is also partly because the supercrystal phase can only be formed when large-scale redistribution of electrical charge is facilitated by ultraviolet illumination.

In the Stoica et al. study, dysprosium scandate $\left(\mathrm{DyScO}_{3}\right)$ single crystals were used as substrates for the growth of intercalated thin film stacks (superlattices) of ferroelectric lead titanate $\left(\mathrm{PbTiO}_{3}\right)$ and incipient ferroelectric strontium titanate $\left(\mathrm{SrTiO}_{3}\right)$. This choice of substrate causes the dipolar structure in the system to become extremely conflicted: coherent epitaxial strain throughout the heterostructure favours electrical dipoles in $\mathrm{PbTiO}_{3}$ to orient vertically and those induced in the $\mathrm{SrTiO}_{3}$ layers to be horizontal ${ }^{7}$. This arrangement would, in the absence of mobile charge, be prohibitively energetically costly and so dipolar disruption becomes inevitable. The coupling between polar orientation and strain means that elastic coherence is then threatened and so a complex interplay between competing electrostatic and elastic energy terms results. As might be expected, given the discussion above, periodic superstructures can emerge to ameliorate this conflict. In this case, however, two different superstructures are found to coexist after cooling from the thin film growth temperature: in some regions, $\mathrm{PbTiO}_{3}$ layers form into arrays of clockwise and anticlockwise dipolar columnar vortex pairs (the V-phase), while in others, conventional $90^{\circ}$ ferroelectric-ferroelastic stripe domains (the FEphase) occur (figure 1b).

When exposed to pulses of ultraviolet (UV) light (400nm wavelength), there is a radical unexpected change. The admixture of vortex columns and stripe domains is replaced by a single superstructure of periodically stacked vortex packets within the $\mathrm{PbTiO}_{3}$ layers, accompanied by $\mathrm{SrTiO}_{3}$ layers in which dipoles barely persist (figure $1 \mathrm{~b}$ ). This new superstructure (or S-phase) possesses periodically distributed dipolar discontinuities, which are screened by the free carriers released by the UV illumination (neatly dealt with in phase-field modelling, where posited free energy expressions guide simulated microstructural evolution, by imposing an artificially high matrix permittivity). The 3D periodicity and complexity of the new supercrystal, as revealed by X-ray reciprocal space mapping, is breath-taking (Figure 1c).

Apart from the novelty and aesthetic appeal of the work, its real importance is that it prompts an uncomfortable discussion concerning equilibrium in ferroelectrics. At this point, an important subtlety in the physics of microstructural development must be highlighted: many of the complex periodic or pseudo-periodic microstructures known in materials science do not reflect equilibrium at all. In exsolved eutectics, for example, the phases present and their relative proportions can be rationalised using phase diagrams, but their fine-scale periodic distribution arises because complete long-range equilibrium phase segregation is compromised by limiting kinetics. The equilibrium total segregation state is not given enough time to fully develop. This is not generally the case for periodic superstructures, such as conventional domain patterns, in ferroelectrics. Instead, for these microstructures, a genuine equilibrium exists in which long-range energy costs, typically due to depolarising fields and macroscopic strain in the system, are perfectly balanced by shortrange energy costs, associated with domain walls. Reassurance that such equilibria are genuinely achieved can be seen through the enduring validity of the Landau-Lifshitz-Kittel scaling law in predicting domain periodicities in ferroelectric, ferromagnetic and ferroelastic materials $3,4,8,9$.

An interpretation of the Stoica et al. study is that the true equilibrium superperiodic state (their supercrystal) might only have been accessed when a complete redistribution of charges was facilitated by UV exposure. Without above band-gap illumination, charges confined within conventional bonds in the constituent dielectrics may have been 
prevented from finding potentially lower energy locations at which they could screen dipolar discontinuities, forcing a metastable mish-mash of two non-equilibrium superstructure phases to evolve (the FE and $\mathrm{V}$ phase mixture). The definite and significant reduction in modulated strain and appearance of beautiful Laue fringes in the X-ray characterisation after the UV-induced transition to the supercrystal state, as well as the results from free energy calculations associated with phase-field simulations, point distinctly towards this possibility. Future research into similar supercrystal formation might test this by targeting other ways to facilitate electrostatic screening, such as the intercalation of metallic layers in the superlattice stack.

The disturbing implication of the work by Stoica et al. is that all phase diagrams in ferroelectrics, in which equilibrium superstructure formation is considered, might only be accurately accessed and predicted when complete long-range charge redistribution is facilitated and considered. If this were to be the case, then conventional thermal mapping of phase space would be incapable of tracking true equilibrium behaviour, demanding a rethink of how phase diagrams are obtained in materials, particularly those containing electrical dipoles.

Centre for Nanostructured Media, School of Mathematics and Physics, Queen's University Belfast, BELFAST, U. K. BT71NN e-mail: M.Gregg@qub.ac.uk

References:

1. S. Getzin et al. Proc. Natl. Acad. Sci. U. S. A. 113, 3551-3556 (2016).

2. C. E. Tarnita et al. Nature 541, 398-401 (2017).

3. L. Landau, E. Lifshitz, Phys, Z. Sowjetunion 8, 153-164 (1935).

4. C. Kittel, Phys. Rev. 70, 965-971 (1946).

5. V. A. Stoica et al. Nat. Mater. (2019).

6. A. K. Yadav et al. Nature 530, 198-201 (2016).

7. J. H. Haeni et al. Nature 430, 758-761 (2004).

8. T. Mitsui, J. Furuichi, Phys. Rev. 90, 193-201 (1953).

9. A. L. Roytburd, Phys. Status Solidi A 37, 329-339 (1976).

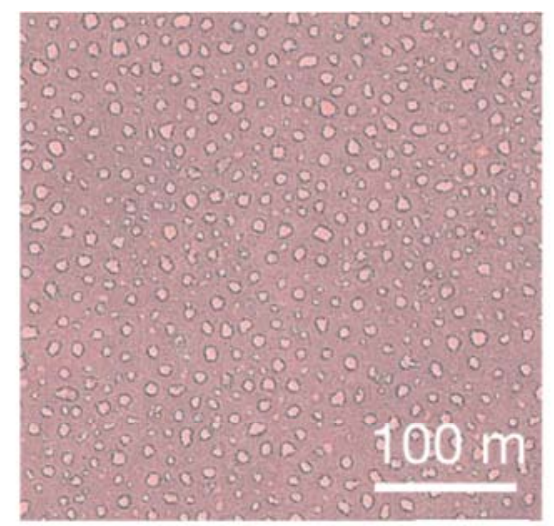

a

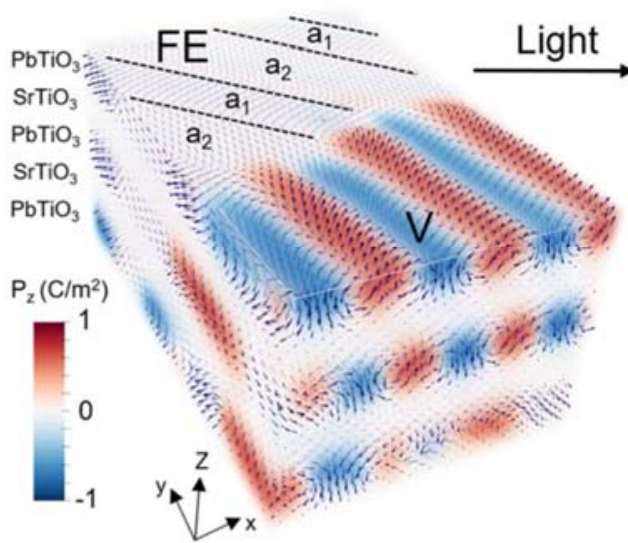

b Coexisting Superstructures

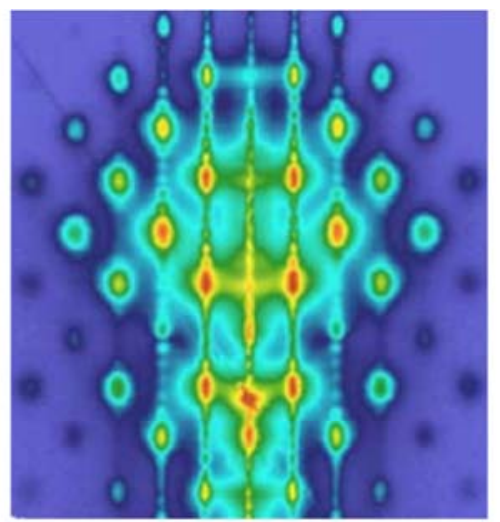

C

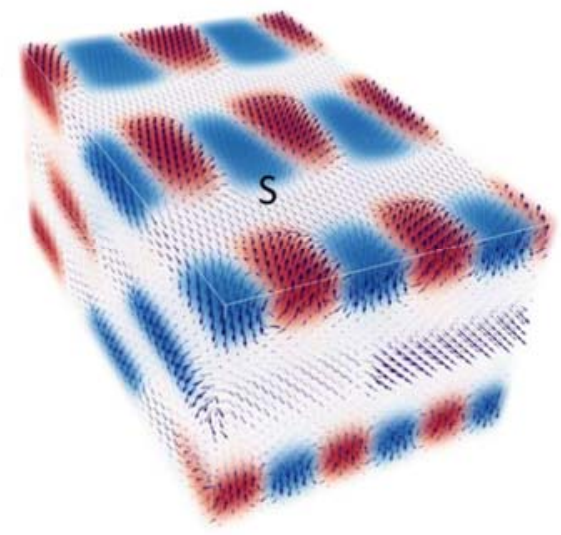

Single-Phase "Supercrystal"

Figure 1 | Periodic superstructures balancing conflicting demands in different situations. a, In the arid grasslands of Namibia and Australia periodic "fairy rings" allow a compromise between the drive for plant life to cover the landscape and limitations associated with environmental factors, such as low rainfall, which put a ceiling on the total biomass which can be supported, $\mathbf{b}$, in ferroelectric heterostructures, "supercrystals" form after ultraviolet illumination to allow minimisation of conflicting long-range and short-range energetics. c, Stunning periodicity in these supercrystals can be seen in reciprocal space maps produced by X-ray scattering.

[Note to the art editor: Figure 1a taken from reference 2 Figure 1f.Figure 1b taken from Figure 1a of reference 5, and Figure $1 c$ taken from Figure $2 b$ of reference 5.$]$ 